\title{
Minimum Deviation Models for Multiple Attribute Decision Making in Intuitionistic Fuzzy Setting
}

\author{
Guiwu Wei* and Xiaofei Zhao \\ Institute of Decision Sciences, Chongqing University of Arts and Sciences \\ Yongchuan 402160, China \\ *Corresponding author,E-mail:weiguiwu@163.com \\ Received: 25-02-2010 \\ Accepted: 11-02-2011
}

\begin{abstract}
With respect to intuitionistic fuzzy multiple attribute decision making problems with preference information on alternatives and incomplete weight information, a method based the minimum deviation is proposed. Firstly, some operational laws of intuitionistic fuzzy numbers, score function and accuracy function of intuitionistic fuzzy numbers are introduced. Then, to reflect the decision maker's preference information, an optimization model based on the minimum deviation method, by which the attribute weights can be determined, is established. For the special situations where the information about attribute weights is completely unknown, we establish another optimization model. By solving this model, we get a simple and exact formula, which can be used to determine the attribute weights. We utilize the intuitionistic fuzzy weighted averaging (IFWA) operator to aggregate the intuitionistic fuzzy information corresponding to each alternative, and then rank the alternatives and select the most desirable one(s) according to the score function and accuracy function. The method can sufficiently utilize the objective information, and meet decision makers' subjective preference, can also be easily performed on computer. Finally, an illustrative example is given to verify the developed approach and to demonstrate its practicality and effectiveness.
\end{abstract}

Key Words: Multiple attribute decision-making; Intuitionistic fuzzy numbers; Intuitionistic fuzzy weighted averaging (IFWA) operator; Weight information, Preference

\section{Introduction}

Atanassov [1-3] introduced the concept of intuitionistic fuzzy set (IFS), which is a generalization of the concept of fuzzy set [4]. The intuitionistic fuzzy set has received more and more attention since its appearance [5-49]. Gau and Buehrer [5] introduced the concept of vague set. But Bustince and Burillo [6] showed that vague sets are intuitionistic fuzzy sets. Chen and Tan [7] presented new techniques for handling multiple attribute fuzzy decision making problems based on vague set theory. And then Hong and Choi [8] provided another technique for handling multiple attribute fuzzy decision making problems based on vague set theory, they provided new functions to measure the degree of accuracy in the grades of membership of each alternative with respect to a set of attribute. However, they assumed that the degree of importance to each attribute is constant. Szmidt and Kacprzyk [9-12] considered the use of intuitionistic fuzzy sets for building soft decision-making models with imprecise information, and proposed two solution concepts about the intuitionistic fuzzy core and the consensus winner for group decision making using intuitionistic fuzzy sets. Szmidt and Kacprzyk [13] proposed a non-probabilistic type of entropy measure for intuitionistic fuzzy sets. Szmidt and Kacprzyk [14] discussed distances between intuitionistic fuzzy sets. Bustince [15] presented different theorems for building intuitionistic fuzzy relations on a set with predetermined 
properties. Li and Cheng [16] studied similarity measures of intuitionistic fuzzy sets and their application to pattern recognitions. Szmidt and Kacprzyk [17] proposed some solution concepts in group decision making with intuitionistic fuzzy preference relations, such as intuitionistic fuzzy core and consensus winner, etc. Szmidt and Kacprzyk [18] investigated the consensus-reaching process in group decision making based on individual intuitionistic fuzzy preference relations. Atanassov et al. [19] provided an algorithm for solving the multi-person multi-attribute decision making problems, in which the attribute weights are given as exact numerical values and the attribute values are expressed in intuitionistic fuzzy numbers. $\mathrm{Li}[20]$ investigated multiple attribute decision making with intuitionistic fuzzy information and constructed several linear programming models to generate optimal weights for attribute. Lin [21] presented a new method for handling multiple attribute fuzzy decision making problems, where the characteristics of the alternatives are represented by intuitionistic fuzzy sets. The proposed method allows the degrees of satisfiability and non-satisfiability of each alternative with respect to a set of attribute to be represented by intuitionistic fuzzy sets, respectively. Furthermore, the proposed method allows the decision-maker to assign the degree of membership and the degree of non-membership of the attribute to the fuzzy concept "importance." Xu [22] investigate the group decision making problems in which all the information provided by the decision makers is expressed as intuitionistic fuzzy decision matrices where each of the elements is characterized by intuitionistic fuzzy number, and the information about attribute weights is partially known, which may be constructed by various forms. $\mathrm{Li}$ [23] extended the linear programming techniques for multidimensional analysis of preference (LINMAP) to develop a new methodology for solving multiattribute decision making problems under Atanassov's intuitionistic fuzzy (IF) environments. Xu and Yager [24] developed some geometric aggregation operators, such as the intuitionistic fuzzy weighted geometric (IFWG) operator, the intuitionistic fuzzy ordered weighted geometric (IFOWG) operator, and the intuitionistic fuzzy hybrid geometric (IFHG) operator and gave an application of the IFHG operator to multiple attribute group decision making with intuitionistic fuzzy information. Xu [25] developed some arithmetic aggregation operators, such as the intuitionistic fuzzy weighted averaging (IFWA) operator, the intuitionistic fuzzy ordered weighted averaging (IFOWA) operator, and the intuitionistic fuzzy hybrid aggregation (IFHA) operator. Xu [26] investigated the intuitionistic fuzzy MADM with the information about attribute weights is incompletely known or completely unknown, a method based on the ideal solution was proposed. Liu and Wang [27] developed an evaluation function for the decision making problem to measure the degrees to which alternatives satisfy and do not satisfy the decision maker's requirement. Then, they proposed the intuitionistic fuzzy point operators, and defined a series of new score functions for the MADM problems based on intuitionistic fuzzy point operators and evaluation function.

In the process of intuitionistic fuzzy MADM with preference information on alternatives, sometimes, the attribute values and preference values on alternatives take the form of intuitionistic fuzzy numbers, and the information about attribute weights is incompletely known or completely unknown because of time pressure, lack of knowledge or data, and the expert's limited expertise about the problem domain. All of the above methods, however, will be unsuitable for dealing with such situations. Therefore, it is necessary to pay attention to this issue. The aim of this paper is to develop a method, based on the minimum deviation method, to overcome this limitation.

The remainder of this paper is set out as follows. In the next section, we introduce some basic concepts related to intuitionistic fuzzy sets. In Section 3 we introduce intuitionistic fuzzy multiple attribute decision making problems with preference information on alternatives, in which the information about attribute weights is incompletely known, and the attribute values and preference values on alternatives take the form of intuitionistic fuzzy numbers. To determine the attribute weights, an optimization model based on the minimum deviation method, by which the attribute weights can be determined, is established. For the special situations where the information about attribute weights is completely unknown, we establish another optimization model. By solving this model, we get a simple and exact formula, which can be used to determine the attribute weights. We utilize the intuitionistic fuzzy weighted averaging 
(IFWA) operator to aggregate the intuitionistic fuzzy information corresponding to each alternative, and then rank the alternatives and select the most desirable one(s) according to the score function and accuracy function. In Section 4, an illustrative example is pointed out. In Section 5 we conclude the paper and give some remarks.

\section{Preliminaries}

In the following, we introduce some basic concepts related to intuitionistic fuzzy sets.

Definition 1 Let $X$ to be a universe of discourse, then a fuzzy set is defined as:

$$
A=\left\{\left\langle x, \mu_{A}(x)\right\rangle \mid x \in X\right\}
$$

which is characterized by a membership function $\mu_{A}: X \rightarrow[0,1]$, where $\mu_{A}(x)$ denotes the degree of membership of the element $x$ to the set $A$ [3].

Atanassov extended the fuzzy set to the IFS, shown as follows:

Definition 2 An IFS $A$ in $X$ is given by

$$
A=\left\{\left\langle x, \mu_{A}(x), v_{A}(x)\right\rangle \mid x \in X\right\}
$$

where $\mu_{A}: X \rightarrow[0,1]$ and $v_{A}: X \rightarrow[0,1]$, with the condition

$$
0 \leq \mu_{A}(x)+v_{A}(x) \leq 1, \quad \forall x \in X
$$

The numbers $\mu_{A}(x)$ and $v_{A}(x)$ represent, respectively, the membership degree and non- membership degree of the element $x$ to the set $A[1,2]$.

Definition 3 For each IFS $A$ in $X$, if

$$
\pi_{A}(x)=1-\mu_{A}(x)-v_{A}(x), \forall x \in X .
$$

Then $\pi_{A}(x)$ is called the degree of indeterminacy of $x$ to $A[1,2]$.

Definition 4 Let $\tilde{a}=(\mu, v)$ be an intuitionistic fuzzy number, a score function $S$ of an intuitionistic fuzzy value can be represented as follows [7]:

$$
S(\tilde{a})=\mu-v, \quad S(\tilde{a}) \in[-1,1] .
$$

Definition 5 Let $\tilde{a}=(\mu, v)$ be an intuitionistic fuzzy number, an accuracy function $H$ of an intuitionistic fuzzy value can be represented as follows [8]:

$$
H(\tilde{a})=\mu+v, \quad H(\tilde{a}) \in[0,1] .
$$

to evaluate the degree of accuracy of the intuitionistic fuzzy value $\tilde{a}=(\mu, v)$, where $H(\tilde{a}) \in[0,1]$. The larger the value of $H(\tilde{a})$, the more the degree of accuracy of the intuitionistic fuzzy value $\tilde{a}$.

As presented above, the score function $S$ and the accuracy function $H$ are, respectively, defined as the difference and the sum of the membership function $\tilde{\mu}_{A}(x)$ and the non-membership function $\tilde{v}_{A}(x) . \mathrm{Xu}$ [24] showed that the relation between the score function $S$ and the accuracy function $H$ is similar to the relation between mean and variance in statistics. Based on the score function $S$ and the accuracy function $H$, in the following, $\mathrm{Xu}$ [24] give an order relation between two intuitionistic fuzzy values, which is defined as follows:

Definition 6 Let $\tilde{a}_{1}=\left(\mu_{1}, v_{1}\right)$ and $\tilde{a}_{2}=\left(\mu_{2}, v_{2}\right)$ be two intuitionistic fuzzy values, $s\left(\tilde{a}_{1}\right)=\mu_{1}-v_{1} \quad$ and $s\left(\tilde{a}_{2}\right)=\mu_{2}-v_{2}$ be the scores of $\tilde{a}$ and $\tilde{b}$, respectively, and let $H\left(\tilde{a}_{1}\right)=\mu_{1}+v_{1} \quad$ and $H\left(\tilde{a}_{2}\right)=\mu_{2}+v_{2}$ be the accuracy degrees of $\tilde{a}$ and $\tilde{b}$, respectively, then if $S(\tilde{a})<S(\tilde{b})$, then $\tilde{a}$ is smaller than $\tilde{b}$, denoted by $\tilde{a}<\tilde{b}$; if $S(\tilde{a})=S(\tilde{b})$, then

(1) if $H(\tilde{a})=H(\tilde{b})$, then $\tilde{a}$ and $\tilde{b}$ represent the same information, denoted by $\tilde{a}=\tilde{b}$; (2) if $H(\tilde{a})<H(\tilde{b}), \tilde{a}$ is smaller than $\tilde{b}$, denoted by $\tilde{a}<\tilde{b}[24]$.

Definition 7 Let $a_{j}=\left(\mu_{j}, v_{j}\right)(j=1,2, \cdots, n)$ be a collection of intuitionistic fuzzy values, and let IFWA: $Q^{n} \rightarrow Q$, if 


$$
\begin{aligned}
& \operatorname{IFWA}_{\omega}\left(\tilde{a}_{1}, \tilde{a}_{2}, \cdots, \tilde{a}_{n}\right)=\sum_{j=1}^{n} \omega_{j} \tilde{a}_{j} \\
& =\left(1-\prod_{j=1}^{n}\left(1-\mu_{j}\right)^{\omega_{j}}, \prod_{j=1}^{n} v_{j}^{\omega_{j}}\right)
\end{aligned}
$$

where $\omega=\left(\omega_{1}, \omega_{2}, \cdots, \omega_{n}\right)^{T}$ be the weight vector of $\tilde{a}_{j}(j=1,2, \cdots, n)$, and $\omega_{j}>0, \sum_{j=1}^{n} \omega_{j}=1$, then IFWA is called the intuitionistic fuzzy weighted averaging (IFWA) operator [25].

Definition 8 Let $\tilde{a}_{1}=\left(\mu_{1}, v_{1}\right)$ and $\tilde{a}_{2}=\left(\mu_{2}, v_{2}\right)$ be two intuitionistic fuzzy numbers, then the normalized Hamming distance between $\tilde{a}_{1}=\left(\mu_{1}, v_{1}\right)$ and $\tilde{a}_{2}=\left(\mu_{2}, v_{2}\right)$ is defined as follows [26]:

$$
d\left(\tilde{a}_{1}, \tilde{a}_{2}\right)=\frac{1}{2}\left(\left|\mu_{1}-\mu_{2}\right|+\left|v_{1}-v_{2}\right|\right)
$$

3 Minimum deviation models for multiple attribute decision making in intuitionistic fuzzy setting with preference information on alternatives

The following assumptions or notations are used to represent the intuitionistic fuzzy MADM problems with incomplete weight information:

(1) The alternatives are known. Let $A=\left\{A_{1}, A_{2}, \cdots, A_{m}\right\}$ be a discrete set of alternatives;

(2) The attributes are known. Let $G=\left\{G_{1}, G_{2}, \cdots, G_{n}\right\}$ be a set of attributes;

(3) The subjective preference information on alternatives is known, and let $\theta=\left(\tilde{\theta}_{1}, \tilde{\theta}_{2}, \cdots, \tilde{\theta}_{m}\right)$ be subjective preference value vector, $\tilde{\theta}_{i}=\left(\alpha_{i}, \beta_{i}\right)$ is intuitionistic fuzzy number, which is subjective preference value on alternative $A_{i}(i=1,2, \cdots, m)$.

(4)The information about attribute weights is incompletely known. Let $w=\left(w_{1}, w_{2}, \cdots, w_{n}\right) \in H$ be the weight vector of attributes, where $w_{j} \geq 0, j=1,2, \cdots, n, \sum_{j=1}^{n} w_{j}=1, H$ is a set of the known weight information, which can be constructed by the following forms [50-53], for $i \neq j$ :

Form 1. A weak ranking: $w_{i} \geq w_{j}$; Form 2. A strict ranking: $w_{i}-w_{j} \geq \alpha_{i}, \alpha_{i}>0$; Form 3. A ranking of differences: $w_{i}-w_{j} \geq w_{k}-w_{l}$, for $j \neq k \neq l$;Form 4 . A ranking with multiples: $w_{i} \geq \beta_{i} w_{j}, 0 \leq \beta_{i} \leq 1$; Form 5. An interval form: $\alpha_{i} \leq w_{i} \leq \alpha_{i}+\varepsilon_{i}, 0 \leq \alpha_{i}<\alpha_{i}+\varepsilon_{i} \leq 1$.

Suppose that $\tilde{R}=\left(\tilde{r}_{i j}\right)_{m \times n}=\left(\mu_{i j}, v_{i j}\right)_{m \times n}$ is the intuitionistic fuzzy decision matrix, where $\mu_{i j}$ indicates the degree that the alternative $A_{i}$ satisfies the attribute $G_{j}$ given by the decision maker, $v_{i j}$ indicates the degree that the alternative $A_{i}$ doesn't satisfy the attribute $G_{j}$ given by the decision maker, $\mu_{i j} \subset[0,1]$, $v_{i j} \subset[0,1] \quad, \quad \mu_{i j}+v_{i j} \leq 1 \quad, \quad i=1,2, \cdots, m \quad$, $j=1,2, \cdots, n$.

Definition 9 Let $\tilde{R}=\left(\tilde{r}_{i j}\right)_{m \times n}=\left(\mu_{i j}, v_{i j}\right)_{m \times n}$ be an intuitionistic fuzzy decision matrix, $\tilde{r}_{i}=\left(\tilde{r}_{i 1}, \tilde{r}_{i 2}, \cdots, \tilde{r}_{i n}\right)$ be the vector of attribute values corresponding to the alternative $A_{i}, i=1,2, \cdots, m$, then we call

$$
\begin{gathered}
\tilde{r}_{i}=\left(\mu_{i j}, v_{i j}\right)=\operatorname{IFWA}_{w}\left(\tilde{r}_{i 1}, \tilde{r}_{i 2}, \cdots, \tilde{r}_{i n}\right) \\
=\left(1-\prod_{j=1}^{n}\left(1-\mu_{i j}\right)^{w_{j}}, \prod_{j=1}^{n} v_{i j}{ }^{w_{j}}\right), \\
i=1,2 \cdot ; \cdot m .
\end{gathered}
$$

the overall value of the alternative $A_{i}$, where $w=\left(w_{1}, w_{2}, \cdots, w_{n}\right)^{T}$ is the weight vector of attributes.

In the situation where the information about attribute weights is completely known, i.e., each attribute weight can be provided by the expert with crisp numerical value, we can weight each attribute value and aggregate all the weighted attribute values corresponding to each 
alternative into an overall one by using Eq. (8). Based on the overall attribute values $\tilde{r}_{i}$ of the alternatives $A_{i}(i=1,2, \cdots, m)$, we can rank all these alternatives and then select the most desirable one(s). The greater $\tilde{r}_{i}$, the better the alternative $A_{i}$ will be.

Because of the complexity of objects, the fuzziness of thought, and the finiteness of knowledge, it' difficult for decision makers to derive the attribute weights, and sometimes, attribute weight information is incompletely known. In this situation, in order to reflect the decision maker's subjective preference and objective information, an optimization model is developed to get the attribute weight. However, there are some differences to the some extent between decision maker's subjective preference and objective information. For the more reasonable decision-making, to select attribute weight vector is to minimize total deviation between objective information and decision maker's subjective preference.

The minimum deviation method is selected here to compute the differences between decision maker's subjective preference and objective information. For the attribute $G_{j} \in G$, the deviation of alternative $A_{i}$ to decision maker's subjective preference can be defined as follows:

$$
D_{i j}(w)=d\left(\tilde{r}_{i j}, \tilde{\theta}_{i}\right) w_{j}, i=1,2, \cdots, m, j=1,2, \cdots, n .
$$

Let

$$
D_{i}(w)=\sum_{j=1}^{n} D_{i j}(w)=\sum_{j=1}^{n} d\left(\tilde{r}_{i j}, \tilde{\theta}_{i}\right) w_{j}, i=1,2, \cdots, m
$$

Then $D_{i}(w)$ represent the deviation value of the alternatives $A_{i}$ to decision maker's subjective preference value $\theta_{i}$.

Based on the above analysis, we have to choose the weight vector $w$ to minimize all deviation values for all the alternatives. To do so, we can construct a linear programming model as follows:

$$
\left\{\begin{array}{l}
\min D(w)=\sum_{i=1}^{m} \sum_{j=1}^{n} D_{i j}(w)=\sum_{i=1}^{m} \sum_{j=1}^{n} d\left(\tilde{r}_{i j}, \tilde{\theta}_{i}\right) w_{j} \\
\text { Subject to } \quad w \in H, \sum_{j=1}^{n} w_{j}=1, w_{j} \geq 0, j=1,2, \cdots, n
\end{array}\right.
$$

where $d\left(\tilde{r}_{i j}, \tilde{\theta}_{i}\right)=\frac{1}{2}\left(\left|\mu_{i j}-\alpha_{i}\right|+\left|v_{i j}-\beta_{i}\right|\right)$.

By solving the model(M-1), we get the optimal solution $w=\left(w_{1}, w_{2}, \cdots, w_{n}\right)$, which can be used as the weight vector of attributes.

If the information about attribute weights is completely unknown, we can establish another programming model:

$(\mathrm{M}-2)$

$$
\left\{\begin{array}{l}
\min D(w)=\sum_{i=1}^{m} \sum_{j=1}^{n} D_{i j}(w)=\frac{1}{2} \sum_{i=1}^{m} \sum_{j=1}^{n}\left(\left|\mu_{i j}-\alpha_{i}\right|+\left|v_{i j}-\beta_{i}\right|\right) w_{j} \\
\text { s.t. } \sum_{j=1}^{n} w_{j}^{2}=1, w_{j} \geq 0, j=1,2, \cdots, n
\end{array}\right.
$$

To solve this model, we construct the Lagrange function:

$$
L(w, \lambda)=\frac{1}{2} \sum_{i=1}^{m} \sum_{j=1}^{n}\left(\left|\mu_{i j}-\alpha_{i}\right|+\left|v_{i j}-\beta_{i}\right|\right) w_{j}+\frac{\lambda}{4}\left(\sum_{j=1}^{n} w_{j}^{2}-1\right)
$$

where $\lambda$ is the Lagrange multiplier.

Differentiating Eq. (9) with respect to $w_{j}(j=1,2, \cdots, n)$ and $\lambda$, and setting these partial derivatives equal to zero, the following set of equations is obtained:

$$
\left\{\begin{array}{l}
\frac{\partial L}{\partial w_{j}}=\sum_{i=1}^{m}\left(\left|\mu_{i j}-\alpha_{i}\right|+\left|v_{i j}-\beta_{i}\right|\right)+\lambda w_{j}=0 \\
\frac{\partial L}{\partial \lambda}=\sum_{j=1}^{n} w_{j}^{2}-1=0
\end{array}\right.
$$

By solving Eq. (10), we get a simple and exact formula for determining the attribute weights as follows:

$$
w_{j}^{*}=\frac{\sum_{i=1}^{m}\left(\left|\mu_{i j}-\alpha_{i}\right|+\left|v_{i j}-\beta_{i}\right|\right)}{\sqrt{\sum_{j=1}^{n}\left[\sum_{i=1}^{m}\left(\left|\mu_{i j}-\alpha_{i}\right|+\left|v_{i j}-\beta_{i}\right|\right)\right]^{2}}}
$$


By normalizing $w_{j}^{*}(j=1,2, \cdots, n)$ be a unit, we have

$$
w_{j}=\frac{\sum_{i=1}^{m}\left(\left|\mu_{i j}-\alpha_{i}\right|+\left|v_{i j}-\beta_{i}\right|\right)}{\sum_{j=1}^{n} \sum_{i=1}^{m}\left(\left|\mu_{i j}-\alpha_{i}\right|+\left|v_{i j}-\beta_{i}\right|\right)}
$$

Based on the above models, we develop a practical method for solving the MADM problems with preference information on alternatives, in which the information about attribute weights is incompletely known or completely unknown, and the attribute values and preference values on alternatives take the form of intuitionistic fuzzy information. The method involves the following steps:

Step 1. Let $\tilde{R}=\left(\tilde{r}_{i j}\right)_{m \times n}$ be an intuitionistic fuzzy decision matrix, where $\tilde{r}_{i j}=\left(\mu_{i j}, v_{i j}\right)$, which is an attribute value, given by an expert, for the alternative $A_{i} \in A$ with respect to the attribute $G_{j} \in G$, $w=\left(w_{1}, w_{2}, \cdots, w_{n}\right)$ be the weight vector of attributes, where $w_{j} \in[0,1], j=1,2, \cdots, n, \quad H$ is a set of the known weight information, which can be constructed by the forms $1-5$. let $\theta=\left(\tilde{\theta}_{1}, \tilde{\theta}_{2}, \cdots, \tilde{\theta}_{m}\right)$ be subjective preference value, $\tilde{\theta}_{i}=\left(\alpha_{i}, \beta_{i}\right)$ is intuitionistic fuzzy number, which is subjective preference value on alternative $A_{i}(i=1,2, \cdots, m)$.

Step 2. If the information about the attribute weights is partly known, then we solve the model (M-1) to obtain the attribute weights. If the information about the attribute weights is completely unknown, then we can obtain the attribute weights by using Eq. (11).

Step 3. Utilize the weight vector $w=\left(w_{1}, w_{2}, \cdots, w_{n}\right)$ and by Eq. (8), we obtain the overall values $\tilde{r}_{i}$ of the alternative $A_{i}(i=1,2, \cdots, m)$.

Step 4. calculate the scores $S\left(\tilde{r}_{i}\right)$ of the overall intuitionistic fuzzy preference value $\tilde{r}_{i}(i=1,2, \cdots, m)$ to rank all the alternatives $A_{i}(i=1,2, \cdots, m)$ and then to select the best one(s) (if there is no difference between two scores $S\left(\tilde{r}_{i}\right)$ and $S\left(\tilde{r}_{j}\right)$, then we need to calculate the accuracy degrees $H\left(\tilde{r}_{i}\right)$ and $H\left(\tilde{r}_{j}\right)$ of the overall intuitionistic fuzzy preference value $\tilde{r}_{i}$ and $\tilde{r}_{j}$, respectively, and then rank the alternatives $A_{i}$ and $A_{j}$ in accordance with the accuracy degrees $H\left(\tilde{r}_{i}\right)$ and $H\left(\tilde{r}_{j}\right)$

Step 5. Rank all the alternatives $A_{i}(i=1,2, \cdots, m)$ and select the best one(s) in accordance with $S\left(\tilde{r}_{i}\right)$ and $H\left(\tilde{r}_{i}\right)(i=1,2, \cdots, m)$.

Step 6. End.

\section{Illustrative Example}

Let us suppose there is an investment company, which wants to invest a sum of money in the best option (adapted from [54-60]). There is a panel with five possible alternatives to invest the money: (1) $\mathrm{A}_{1}$ is a car company; (2) $\mathrm{A}_{2}$ is a food company; (3) $\mathrm{A}_{3}$ is a computer company; (4) $\mathrm{A}_{4}$ is an arms company; (5) $\mathrm{A}_{5}$ is a TV company. The investment company must take a decision according to the following four attributes: (1) $\mathrm{G}_{1}$ is the risk analysis; (2) $\mathrm{G}_{2}$ is the growth analysis; (3) $\mathrm{G}_{3}$ is the social-political impact analysis; (4) $\mathrm{G}_{4}$ is the environmental impact analysis. The five possible alternatives $A_{i}(i=1,2, \cdots, 5)$ are to be evaluated using the intuitionistic fuzzy information by the decision maker under the above four attributes, as listed in the following matrix.

$\tilde{R}=\left[\begin{array}{cccc}(0.4,0.5) & (0.3,0.6) & (0.6,0.3) & (0.7,0.2) \\ (0.3,0.7) & (0.7,0.2) & (0.7,0.2) & (0.4,0.5) \\ (0.4,0.6) & (0.5,0.1) & (0.5,0.3) & (0.6,0.3) \\ (0.4,0.3) & (0.6,0.3) & (0.3,0.4) & (0.2,0.6) \\ (0.2,0.6) & (0.7,0.3) & (0.7,0.1) & (0.5,0.3)\end{array}\right]$ Decision maker's subjective preference values on 
alternative $A_{i}(i=1,2,3,4,5)$ are as follows:

$$
\begin{aligned}
& \tilde{\theta}_{1}=(0.3,0.5), \tilde{\theta}_{2}=(0.6,0.2), \tilde{\theta}_{3}=(0.5,0.4) \\
& \tilde{\theta}_{4}=(0.7,0.2), \tilde{\theta}_{5}=(0.4,0.3)
\end{aligned}
$$

Then, we utilize the approach developed to get the most desirable alternative(s).

Case 1: The information about the attribute weights is partly known and the known weight information is given as follows:

$$
\begin{gathered}
H=\left\{0.20 \leq w_{1} \leq 0.30,0.10 \leq w_{2} \leq 0.15,\right. \\
0.20 \leq w_{3} \leq 0.28,0.30 \leq w_{4} \leq 0.35, \\
\left.w_{j} \geq 0, j=1,2,3,4, \sum_{j=1}^{4} w_{j}=1\right\}
\end{gathered}
$$

Step 1. Utilize the model (M-1) to establish the following single-objective programming model:

$$
\left\{\begin{array}{l}
\min D(w)=1.05 w_{1}+0.50 w_{2}+0.90 w_{3}+1.20 w_{4} \\
\text { s.t. } w \in H
\end{array}\right.
$$

Solving this model, we get the weight vector of attributes:

$$
w=\left(\begin{array}{llll}
0.2700 & 0.1500 & 0.2800 & 0.3000
\end{array}\right)^{T}
$$

Step 2. Utilize the weight vector $w$ and by Eq. (8), we obtain the overall values $\tilde{r}_{i}$ of the alternatives $A_{i}(i=1,2,3,4,5)$.

$$
\begin{aligned}
& \tilde{r}_{1}=(0.5548,0.3383), \tilde{r}_{2}=(0.5357,0.3692) \\
& \tilde{r}_{3}=(0.5088,0.3068), \tilde{r}_{4}=(0.3574,0.4003) \\
& \tilde{r}_{5}=(0.5443,0.2660)
\end{aligned}
$$

Step 3. Calculate the scores $S\left(\tilde{r}_{i}\right)$ of the overall intuitionistic fuzzy preference values $\tilde{r}_{i}(i=1,2,3,4,5)$

$$
\begin{aligned}
& S\left(\tilde{r}_{1}\right)=0.2164, S\left(\tilde{r}_{2}\right)=0.1665 \\
& S\left(\tilde{r}_{3}\right)=0.2020, S\left(\tilde{r}_{4}\right)=-0.0430 \\
& S\left(\tilde{r}_{5}\right)=0.2783
\end{aligned}
$$

Step 4. Rank all the alternatives $A_{i}(i=1,2,3,4,5)$ in accordance with the scores $S\left(\tilde{r}_{i}\right) \quad(i=1,2, \cdots, 5)$ of the overall intuitionistic fuzzy preference values $\tilde{r}_{i}(i=1,2,3,4,5): A_{5} \succ A_{1} \succ A_{3} \succ A_{2} \succ A_{4}$, and thus the most desirable alternative is $A_{5}$.

Case 2: If the information about the attribute weights is completely unknown, we utilize another approach developed to get the most desirable alternative(s).

Step 1. Utilize the Eq. (11) to get the weight vector of attributes:

$$
w=\left(\begin{array}{llll}
0.2877 & 0.1370 & 0.2465 & 0.3288
\end{array}\right)^{T}
$$

Step 2. Utilize the weight vector $w$ and by Eq. (8), we obtain the overall values $\tilde{r}_{i}$ of the alternatives $A_{i}(i=1,2,3,4,5)$.

$$
\begin{aligned}
& \tilde{r}_{1}=(0.5585,0.3344), \tilde{r}_{2}=(0.5192,0.3876) \\
& \tilde{r}_{3}=(0.5103,0.3150), \tilde{r}_{4}=(0.3519,0.4045) \\
& \tilde{r}_{5}=(0.5294,0.2793)
\end{aligned}
$$

Step 3. Calculate the scores $S\left(\tilde{r}_{i}\right)(i=1,2,3,4,5)$ of the overall intuitionistic fuzzy preference values $\tilde{r}_{i}(i=1,2,3,4,5)$

$$
\begin{aligned}
& S\left(\tilde{r}_{1}\right)=0.2241, S\left(\tilde{r}_{2}\right)=0.1316 \\
& S\left(\tilde{r}_{3}\right)=0.1953, S\left(\tilde{r}_{4}\right)=-0.0525 \\
& S\left(\tilde{r}_{5}\right)=0.2501
\end{aligned}
$$

Step 4. Rank all the alternatives $A_{i}(i=1,2,3,4,5)$ in accordance with the scores $S\left(\tilde{r}_{i}\right)$ : $A_{5} \succ A_{1} \succ A_{3} \succ A_{2} \succ A_{4}$, and thus the most desirable alternative is $A_{5}$.

\section{Conclusion}

In this paper, we have investigated intuitionistic fuzzy multiple attribute decision making problems with preference information on alternatives, in which the information about attribute weights is incompletely known, and the attribute values and preference values on alternatives take the form of intuitionistic fuzzy numbers. To determine the attribute weights, an optimization model based on the minimum deviation method, by which make use of the subjective information provided by the decision 
maker and the known objective information, is established to derive the attribute weights. We utilize the intuitionistic fuzzy weighted averaging (IFWA) operator to aggregate the intuitionistic fuzzy information corresponding to each alternative, and then rank the alternatives and select the most desirable one(s) according to the score function and accuracy function. Thus, the resultant ranking of alternative reflects both the objective information and the decision maker's subjective considerations. Finally, an illustrative example is given. Furthermore, we can also extend the developed models and procedures to deal with the MADM with interval-valued intuitionistic fuzzy information. In future research, our work will focus on the application of intuitionistic fuzzy multiple attribute decision making in the fields such as investment, personnel examination, medical diagnosis, and military system efficiency evaluation.

\section{Acknowledgment}

This research was supported by the Humanities and Social Sciences Foundation of Ministry of Education of the People's Republic of China under Grant No.09XJA630010 and the China Postdoctoral Science Foundation under Grant 20100480269.

\section{References}

[1] K. Atanassov, Intuitionistic fuzzy sets, Fuzzy Sets and Systems 20 (1986) 87-96.

[2] K. Atanassov, More on intuitionistic fuzzy sets, Fuzzy Sets and Systems 33 (1989) 37- 46.

[3] K. Atanassov, Two theorems for intuitionistic fuzzy sets, Fuzzy Sets and Systems 110 (2000) 267-269.

[4] L. A. Zadeh, Fuzzy sets, Information and Control 8 (1965)338-356.

[5] W. L. Gau and D. J. Buehrer, Vague sets, IEEE Transactions on Systems, Man and Cybernetics 23 (2) (1993) 610-614.

[6]H.Bustine and P. Burillo, Vague sets are intuitionistic fuzzy sets, Fuzzy Sets and Systems 79 (1996) 403-405.

[7] S.M. Chen and J.M. Tan, Handling multicriteria fuzzy decision-making problems based on vague set theory, Fuzzy Sets Systems 67 (1994) 163-172.

[8] D. H. Hong and C.H. Choi, Multicriteria fuzzy decision-making problems based on vague set theory,
Fuzzy Sets Systems 114 (2000) 103-113.

[9] E. Szmidt and J. Kacprzyk, Intuitionistic fuzzy sets in group decision making, NIFS 2 (1) (1996) 15-32.

[10]E. Szmidt and J. Kacprzyk, Remarks on some applications of intuitionistic fuzzy sets in decision making, NIFS 2 (3) (1996) 22-31.

[11] E. Szmidt and J. Kacprzyk, Group decision making via intuitionistic fuzzy sets, FUBEST'96, Sofia, Bulgaria, October 9-11, 1996, pp. 107-112.

[12] E. Szmidt and J. Kacprzyk, Intuitionistic fuzzy sets for more realistic group decision making, International Conference Transition to Advanced Market Institutions and Economies,Warsaw, June 18-21, 1997, pp. 430-433.

[13]E. Szmidt and J. Kacprzyk, Entropy for intuitionistic fuzzy sets, Fuzzy Sets and Systems 118 (2001) 467-477.

[14]E. Szmidt and J. Kacprzyk, Distances between intuitionistic fuzzy sets, Fuzzy Sets and Systems 114 (2001) 505-518.

[15] H. Bustince, Construction of intuitionistic fuzzy relations with predetermined properties, Fuzzy Sets and Systems 109 (2000) 379-403.

[16]D.F. Li and C. T. Cheng, New similarity measures of intuitionistic fuzzy sets and application to pattern recognitions, Pattern Recognition Letters 23 (1-3) (2002) 221-225.

[17]E. Szmidt and J. Kacprzyk, Using intuitionistic fuzzy sets in group decision making, Control and Cybernetics 31 (2002) 1037-1053.

[18]E. Szmidt and J. Kacprzyk, A consensus-reaching process under intuitionistic fuzzy preference relations. International Journal of Intelligent Systems 18 (2003) 837-852.

[19]K. Atanassov, G. Pasi and R. R. Yager, Intuitionistic fuzzy interpretations of multi-criteria multiperson and multi-measurement tool decision making, International Journal of Systems Science 36 (2005) 859-868.

[20] D.F. Li, Multiattribute decision making models and methods using intuitionistic fuzzy sets, Journal of Computer and System Sciences 70 (2005) 73-85.

[21] L. Lin, X. H. Yuan and Z.Q. Xia, Multicriteria fuzzy decision-making methods based on intuitionistic fuzzy sets, Journal of Computer and System Sciences 73 (2007) 84-88.

[22]Z. S. Xu, Multi-person multi-attribute decision making models under intuitionistic fuzzy environment, Fuzzy Optimization and Decision Making 6(3) (2007) 
221-236.

[23] D.F. Li, Extension of the LINMAP for multiattribute decision making under Atanassov's intuitionistic fuzzy environment, Fuzzy Optimization and Decision Making 7 (1) (2008)17-34

[24]Z. S. Xu and R. R. Yager, Some geometric aggregation operators based on intuitionistic fuzzy sets, International Journal of General System 35 (2006) 417-433.

[25] Z. S. Xu, Intuitionistic fuzzy aggregation operators, IEEE Transactions on Fuzzy Systems 15(6) (2007) 1179-1187.

[26]Z. S. Xu, Models for multiple attribute decision-making with intuitionistic fuzzy information, International Journal of Uncertainty, Fuzziness and Knowledge-Based Systems 15(3) (2007) 285-297.

[27] H.W. Liu, G.J. Wang, Multi-criteria decision-making methods based on intuitionistic fuzzy sets, European Journal of Operational Research 179 (2007) 220-233.

[28] G. W. Wei, Induced intuitionistic fuzzy ordered weighted averaging operator and its application to multiple attribute group decision making. Lecture Notes in Artificial Intelligence 5009(2008)124-131.

[29]D. F. Li, Some measures of dissimilarity in intuitionistic fuzzy structures, Journal of Computer and systems Sciences 68 (1) (2004) 115-122.

[30]D.F. Li, Y.C.Wang, S. Liu, F. Shan, Fractional programming methodology for multi-attribute group decision making using IFS, Applied Soft Computing Journal 9 (2009) 219-225.

[31]G.W.Wei,Maximizing deviation method for multiple attribute decision making in intuitionistic fuzzy setting, Knowledge-based Systems 21(8) (2008) 833-836.

[32]G.W. Wei, GRA method for multiple attribute decision making with incomplete weight information in intuitionistic fuzzy setting, Knowledge-based Systems 23(3) (2010) 243-247.

[33]G.W. Wei, Some geometric aggregation functions and their application to dynamic multiple attribute decision making in intuitionistic fuzzy setting, International Journal of Uncertainty, Fuzziness and Knowledge-Based Systems 2009 17(2) (2009) 179-196.

[34] Z. S. Xu, Dynamic intuitionistic fuzzy multi-attribute decision making, International Journal of Approximate Reasoning 48(1) (2008) 246-262.

[35] H. Zhao, Z.S. Xu, M.F. Ni and S.S. Liu, Generalized aggregation operators for intuitionistic fuzzy sets,
International Journal of Intelligent System 25(1) (2010) 1-30.

[36]Z.S. Xu and X.Q. Cai, Recent advances in intuitionistic fuzzy information aggregation,Fuzzy Optimization and Decision Making, 9(4) (2010) 359-381.

[37]Z.S. Xu, Choquet integrals of weighted intuitionistic fuzzy information. Information Sciences, 180 (2010) 726-736.

[38]Z.S.Xu and R.R.Yager, Intuitionistic and interval-valued intutionistic fuzzy preference relations and their measures of similarity for the evaluation of agreement within a group, Fuzzy Optimization Decision Making 8(2) (2009) 123-139.

[39]X.F.Wang, Fuzzy number intuitionistic fuzzy arithmetic aggregation operators, International Journal of Fuzzy Systems 10(2) (2008) 104-111.

[40] G. W. Wei, X.F. Zhao, H.J. Wang and R. Lin, GRA model for selecting an ERP system in trapezoidal intuitionistic fuzzy setting, Information: An International Interdisciplinary Journal 13(4) (2010) 1143-1148.

[41] G. W. Wei, X.F. Zhao, R. Lin. Some induced aggregating operators with fuzzy number intuitionistic fuzzy information and their applications to group decision making, International Journal of Computational Intelligence Systems 3(1) (2010) 84-95.

[42] G. W. Wei, R. Lin, X.F. Zhao and H.J. Wang, TOPSIS-based linear-programming methodology for multiple attribute decision making with incomplete weight information in intuitionistic fuzzy setting, Information: An International Interdisciplinary Journal 13(5) (2010).

[43] G. W. Wei, H.J. Wang, R. Lin, Application of correlation coefficient to interval-valued intuitionistic fuzzy multiple attribute decision making with incomplete weight information, Knowledge and Information Systems 26(2) (2011) 337-349.

[44] G. W. Wei, Some induced geometric aggregation operators with intuitionistic fuzzy information and their application to group decision making, Applied Soft Computing 10(2) (2010) 423-431.

[45] T.R. Li, D. Ruan, W. Geert, J. Song, Y. Xu, A rough sets based characteristic relation approach for dynamic attribute generalization in data mining, Knowledge-Based Systems 20(5) (2007) 485-494.

[46]L. Martínez, J. Liu, D. Ruan, J.B. Yang, Dealing with 
heterogeneous information in engineering evaluation processes, Information Sciences 177(7) (2007) 1533-1542.

[47]X.B. Li, D. Ruan, J. Liu and Y. Xu, A linguistic-valued weighted aggregation operator to multiple attribute group decision making with quantative and qualitative information, International Journal of Computational Intelligence Systems 1(3) (2008) 274-284.

[48] J. M. Merigó, A. M. Gil-Lafuente, The induced generalized OWA operator, Information Sciences 179(6) (2009) 729-741.

[49] C. Kahraman, A. Cagri Tolga, An alternative ranking approach and its usage in multi-criteria decision-making, International Journal of Computational Intelligence Systems 2(3) (2009) 219-235.

[50] K.S. Park and S.H. Kim, Tools for interactive multi-attribute decision making with incompletely identified information, European Journal of Operational Research 98 (1997) 111-123.

[51] S.H. Kim, S.H. Choi and J.K. Kim, An interactive procedure for multiple attribute group decision making with incomplete information: range-based approach, European Journal of Operational Research 118 (1999) 139-152.

[52] S.H. Kim and B.S. Ahn, Interactive group decision making procedure under incomplete information, European Journal of Operational Research 116 (1999) 498-507.

[53] K.S. Park, Mathematical programming models for charactering dominance and potential optimality when multicriteria alternative values and weights are simultaneously incomplete. IEEE transactions on systems, man, and cybernetics-part A, Systems and Humans, 34 (2004) 601-614.

[54] F. Herrera and E. Herrera-Viedma, Linguistic decision analysis: steps for solving decision problems under linguistic information, Fuzzy Sets and Systems 115 (2000) 67-82.

[55]G. W. Wei, A method for multiple attribute group decision making based on the ET-WG and ET-OWG operators with 2-tuple linguistic information, Expert Systems with Applications 37(12)(2010) 7895-7900.

[56]G. W. Wei, R. Lin, X.F. Zhao, H.J. Wang, Models for multiple attribute group decision making with 2-tuple linguistic assessment information, International Journal of Computational Intelligence Systems 3(3)
(2010) 315-324.

[57]G. W. Wei, Uncertain linguistic hybrid geometric mean operator and its Application to group decision making under uncertain linguistic environment, International Journal of Uncertainty, Fuzziness and Knowledge-Based Systems 17(2) (2009) 251-267.

[58] G. W. Wei, Grey relational analysis method for 2-tuple linguistic multiple attribute group decision making with incomplete weight information, Expert Systems with Applications 38 (5) (2011) 4824-4828.

[59]G. W. Wei, FIOWHM operator and its application to multiple attribute group decision making, Expert Systems with Applications 38 (4) (2011) 2984-2989

[60]G. W. Wei, Extension of TOPSIS method for 2-tuple linguistic multiple attribute group decision making with incomplete weight information, Knowledge and Information Systems 25 (2010) 623-634.

[61]G. W. Wei, A novel efficient approach for interval-valued intuitionistic fuzzy multiple attribute decision making with incomplete weight information, Information: An International Interdisciplinary Journal 14(1) (2011) 97-102. 